\title{
Migration of the ICS Local Terrestrial Reference System to RRS04
}

\author{
Mamadou N. Faye, Diogoye Diouf \\ Unité de Formation et de Recherche-Sciences de l’Ingénieur, Université Iba Der Thiam de Thiès, Thiès, Sénégal \\ Email: mdiaye.faye@univ-thies.sn
}

How to cite this paper: Faye, M.N. and Diouf, D. (2021) Migration of the ICS Local Terrestrial Reference System to RRS04. International Journal of Geosciences, 12, 689-703. https://doi.org/10.4236/ijg.2021.128039

Received: June 29, 2021

Accepted: August 20, 2021

Published: August 23, 2021

Copyright $\odot 2021$ by author(s) and Scientific Research Publishing Inc. This work is licensed under the Creative Commons Attribution International License (CC BY 4.0).

http://creativecommons.org/licenses/by/4.0/

\begin{abstract}
In order to comply with the recommendations of the new mining code, as well as to facilitate data acquisition and exchange, and to guarantee the reliability of these data, the Chemical Industries of Senegal (named ICS) have decided to switch from their local terrestrial reference system to the Senegalese terrestrial reference system created in 2004 and called RRS04. This move allows ICS to take advantage of new measurement techniques such as GNSS and a more global terrestrial reference system. A combination of geodesy with the least squares method was implemented for the resolution of the problem and the optimization of the solution. For this purpose, a GNSS observation campaign was carried out on selected points. The raw data obtained were processed with the Leica Infinity 3.0 software before being used in our calculations for the estimation of the parameters of this passage which has many stakes for the ICS. The obtained results were then presented, applied before being checked and validated.
\end{abstract}

\section{Keywords}

Topography, Coordinate System Transformation, Migration, ICS, RRS04

\section{Introduction}

In the absence of a national reference system, Senegal has known before and during the 20th century, the realization of different local datum. Entities, companies and organizations had to set up a local coordinate system to ensure their topographic work. The mining sector was not an exception to the rule and that is why the ICS have had their own coordinate system for decades for the planning and monitoring of their extractive activities. It was only in 2004 that Senegal created and adopted a national terrestrial reference system called RRS04 with 20 first-order points, which were only reinforced in 2008 by 137 other second-order points. Sub- 
sequently, as part of the modernization of the mining sector, the State of Senegal has undertaken reforms including the standardization of the sector's terrestrial reference systems by requiring the adoption of RRS04 as the terrestrial reference system for spatial data by the mining industries. In addition to this recommendation by the authorities, the ICS decided to leave their local terrestrial reference system, which was faced with, among other things, incomplete documentation, lack of knowledge of certain characteristics, and limited coverage for Senegalese terrestrial reference system (RRS04).

It should also be noted that the local coordinate system in use is not compatible enough with modern Global Navigation Satellite System (GNSS) measurement technology to allow some work with the GNSS receiver. It is clear that nowadays the requirements for accuracy and data quality cannot be guaranteed by a local coordinate system, realized by instruments and methods of classical geodesy, outdated by today's technical progress. Thus, with the advent of space geodesy, the need to work in global systems more accurate and offering homogeneity of reference systems and processed data is required.

The main objective of this work is to determine the transformation parameters between the two systems for the adaptation of the ICS local terrestrial reference system to Senegalese national terrestrial reference system.

The methodology used in this work consists of making raw measurements in the field with a GNSS receiver and then processing and exploiting the data obtained.

At the end of this project, the ICS should have a canvas attached to the RRS04 with a worst-case accuracy equal to $\pm 5 \mathrm{~cm}$ which will depend on that of the measurements and that of the existing local terrestrial reference system.

In this article, the context and the stakes of the transition to RRS04, the presentation of the systems in question, the methodology of the transition and the validation of the results obtained are discussed.

\section{Context}

In order to strengthen the attractiveness of the mining sector and to correct certain shortcomings that appeared in Law No. 2003-36 of November 24, 2003 on the mining code, Senegal has embarked since 2013 on a process of reforming the texts that govern the mining sector [1]. This led to the adoption of the law $n^{\circ} 2016$ 32 of November 08, 2016 on the mining code. Article 13 of the implementing decree of this law stipulates that "the perimeter of a research permit is defined by coordinates attached to the national terrestrial reference system (RRS04) or by actual topographic lines (rivers, coasts, state borders) or by a combination of both."

Moreover, the context of industrial and technological internationalization invites the companies of the mining sector as the ICS, to readapt their mode of operation in relation to the techniques, means and methods more global, more modern and more efficient. Thus, with its national and foreign collaborators, the phosphate giant exchanges data constantly and wishes to have a more global and pre- 
cise reference system to guarantee the ease of exchange of mining and geological data as well as the homogeneity and coherence of the latter. Currently, the company has a local coordinate system for the for the monitoring of its mine as well as for the planning and evaluation of operations. This system is the reference the reference of all geographical and geological data of the company at the time when the mining administration in charge of the promotion, regulation, monitoring and control of mining operations recommends the adoption of RRS04 as the standard reference for geological and mining data. This recommendation is intended to standardize the data in the mining cadaster. The mining cadaster is a geological and mining database connected to a GIS that allows the mining administration to produce and update the cartographic representation of mining authorizations and titles and related information [2]. Mining companies must therefore comply with this recommendation to ensure a modern and efficient sector as well as effective political-technical support.

\section{Challenges of the Transition to RRS04}

There are many issues at stake in the transition to RRS04 for the ICS. This project must meet the expectations of the ICS by providing them with an adequate and quality contact system. It must solve the problems of lack of the local system. Indeed, the ICS will have a complete and available documentation on their new datum. They will have a perfect knowledge of all the features of the new system. With the connection, the industry will have a datum with national coverage, associated with the UTM projection. This projection will allow the company to be in phase with the competent control body that wishes to work with this projection. The adoption of RRS04 will allow the company to easily exchange geospatial data within the country but especially abroad. Another challenge of this transition is to allow ICS to take full advantage of the modern GNSS measurement technology for all topographic work to be done.

However, there is a major issue of this transition that corresponds to the redefinition in the RRS04 of all existing spatial data to ICS, without loss and without bias.

\section{Presentation of the Systems Used}

\subsection{The Local Datum}

The exploitation of phosphate quarries in Darou Khoudoss began in the second half of the 1950s (1957) in Ndomor with the Senegalese Company of Phosphates of Taiba (CSPT). It is now managed by the ICS following a merger in 1996 between the CSPT and the ICS themselves. For any mining industry, for the followup of the mine, the evaluation of the exploitations as well as for a good planning of the works, it is imperative to have precise geospatial data expressed in relation to a coordinate system, materialized by a frame of geodetic markers. Thus, the CSPT had set up its own topographic framework to cover the exploitation area of its first deposit located in Ndomor, based on the GEOFFROY framework executed 
in 1958 and on the altimetric markers of the National Geographic Institute (IGN) at that time. Over time, the company was faced with problems such as the disappearance of markers due to mining activities or the non-existence of support points in new exploitation areas. Thus, the CSPT was obliged to reinforce and widen the coverage of its topographic frame. To this end, after the one in Ndomor, a second frame was set up for the exploitation of the Keur Mor Fall (KMF) deposit. Finally, in 1997, ICS established a third frame of benchmarks for the exploitation of the Toben deposit, which is still in progress. All of these benchmark frames define the topographic framework of the ICS, which constitutes the materialization of the local terrestrial reference system (Figure 1).

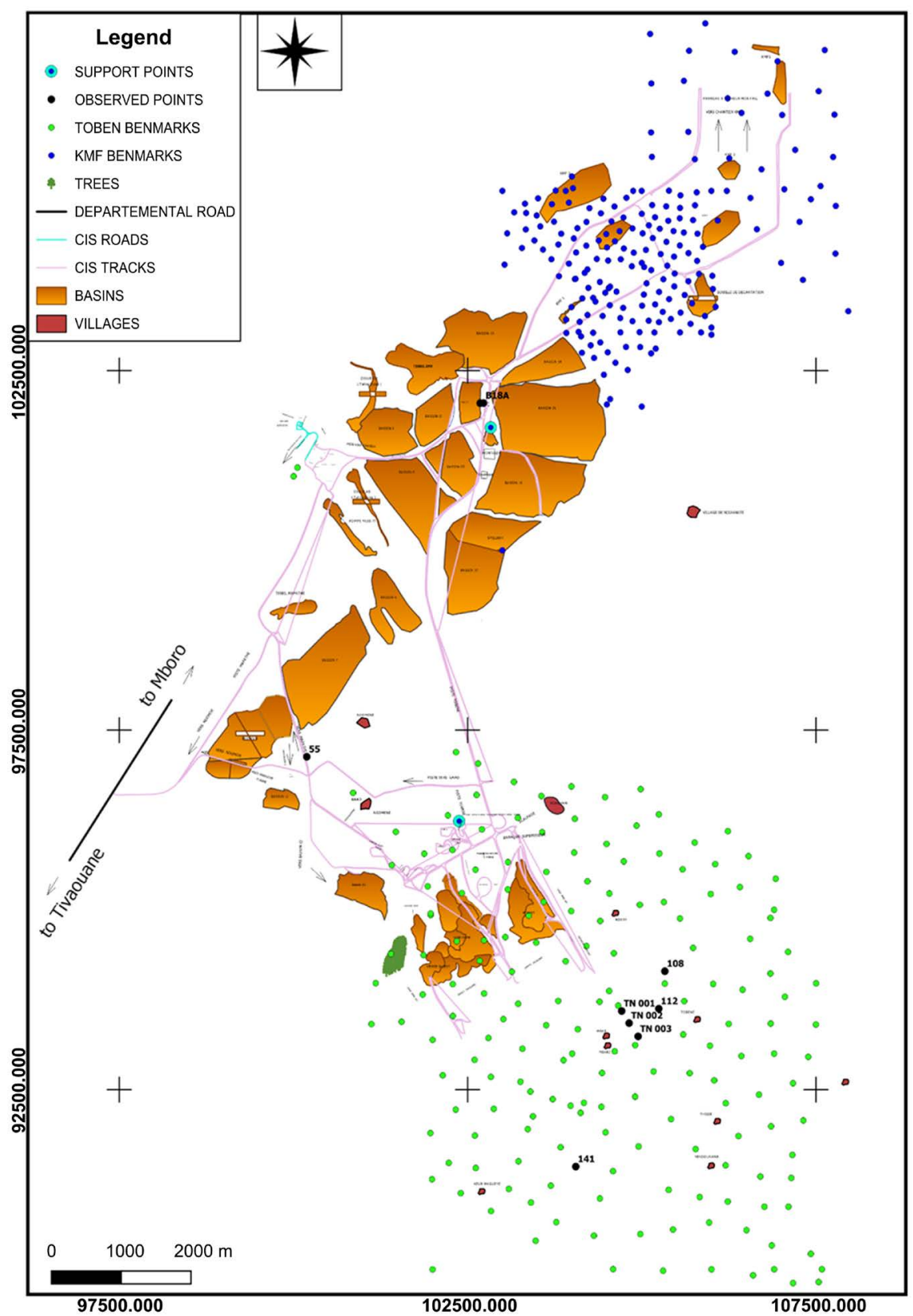

Figure 1. Local terrestrial reference system of the ICS with the observation scheme. 
The topographic equipment used during the KMF and Toben frame development work was of WILD manufacturer and included:

- a total station, the THEOMAT-2000 with an angular accuracy of $1.5 \mathrm{cc}$ and standard deviation of $5 \mathrm{~mm}+5 \mathrm{~mm} / \mathrm{km}$ on distance measurements;

- six prismatic reflectors with telescopic rods and rod holders and a maximum range of $2 \mathrm{~km}$ [3].

At KMF, before beginning his observations, the project manager took the trouble to check the orientation of the bases connecting the support points from the azimuth of the sun by positional astronomy [3]. He also checked the support points before making his measurements. The topographic process consists of a complementary triangulation, followed by a polygonation network and a detail survey. The main paths of this polygonation refer, on average, to four triangulation points each, both at the start and at the finish. The calculations gave an average angular closure of 15 centesimal seconds and an average positional closure of 1 $\mathrm{cm}[3]$.

For altimetry, this is indirect leveling. The leveling is attached trigonometrically and by reciprocal sights to that of BOUETTE. The root mean square error (rms) of the elevations is $2 \mathrm{~mm}$ and the mean closure error is $8 \mathrm{~mm}$ [3].

At Toben, all operations were performed by triangulation. For this reason, the monuments are generally placed on mounds to allow inter-visibility, which is fundamental in triangulation.

\subsection{The RRS04}

During the 20th century, Senegal has had a variety of terrestrial reference systems for specific needs and in limited areas. It is within the framework of the realization of the Senegal mapping project at 1:200,000 scale (PCS200) that the Direction of Geographic and Cartographic Works (DTGC) undertook in 2004, in collaboration with the National Geographic Institute of France (IGN) the implementation of the first national terrestrial reference system named RRS04. This, to allow the homogenization and the standardization of the Senegalese terrestrial reference system as well as its compatibility with the new technologies like GNSS. The RRS04 is a three-dimensional, geocentric frame realized from GPS observations. The coordinates are expressed in the ITRF2000 at epoch 2004.55. The RRS04 is the local realization of the ITRF2000. It includes 20 first-order points established in 2004, covering all Senegalese regions and 137 second-order points established in 2008 and covering all Senegalese departments (Figure 2).

Of these points, 26 are collocated [4]. For the setting of the 20 points of the first order, the calculation was not done in block contrary to the specifications for establishing a geodetic network. A first point, that of Dakar, was first computed from 5 stations of the International GNSS Service (IGS) frame, for attachment to the ITRF2000 epoch 2004.55 then the other 19 points were determined with respect to this first point [5]. This proceeding calculation may affect the internal accuracy of the geodetic frame and its homogeneity. Next, the 137 second-order 


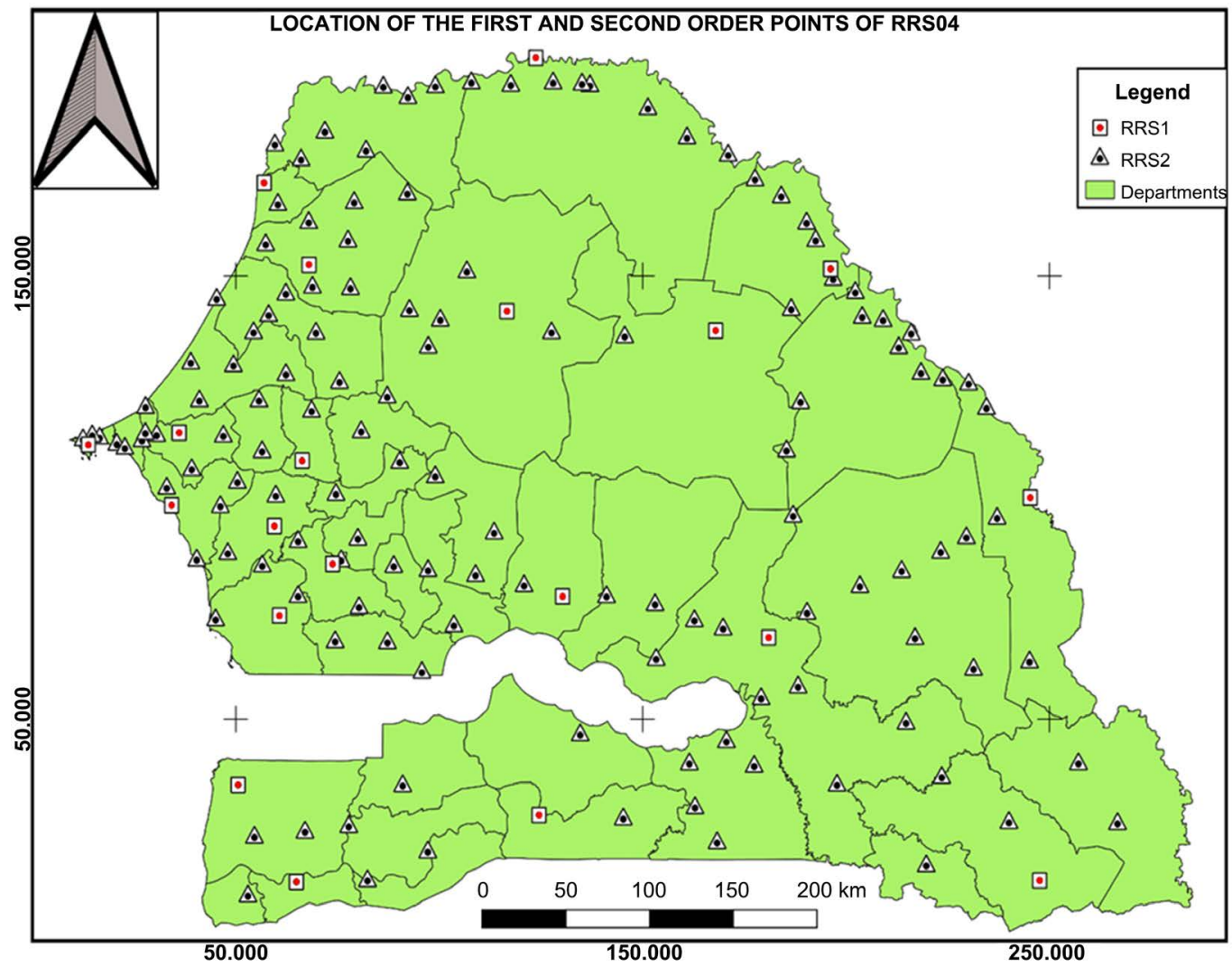

Figure 2. Map of RRS04.

points were established by the cadaster for network densification. Bernese, Trimble Total Control (TTC) and GeoLab software were used for the calculations and compensations of this geodetic frame [5]. However, there is a notable lack of monitoring and maintenance of the network when it is fundamental to preserve the quality of a geodetic frame due to the dynamics of the earth's crust [4].

The characteristics of the network are as follows:

Associated system: ITRF2000, which is the estimation of the International Terrestrial Reference Frame 2000.55 and which has a compatibility of the order of a meter with the world Geodetic system (WGS 84).

Associated ellipsoid: IAG-GRS80 defined by the following parameters [6]:

$$
a=6378137.000 \mathrm{~m}, b=6356752.3141 \mathrm{~m}, 1 / f=298.25722210088
$$

Associated projection: UTM zone 28 North.

Accuracy: The network has a centimetric accuracy.

The accuracies of the coordinates are standard deviations ( 1 sigma values) corresponding to a confidence interval of $39.35 \%$ in planimetry, and $68.3 \%$ on the vertical component [5]. For all points, the semi-major axis of the standard error ellipse never exceeds 1.5 centimeters. The amplitude of the error on the vertical component is everywhere lower than 2 centimeters [5].

On the other hand, there is an absence of information on the level of coherence of the said network [4]. 


\section{GNSS Observation Campaign and Data Processing}

In this work, the device used for the observations is a Leica GPS 1200 receiver and the GNSS computation software used is Leica Infinity, version 3.0.

\subsection{Terminals Prospecting}

For the survey of the pre-selected points for the calculation of the transformation parameters, we had a directory of the topographic markers of the site, a plan in digital format and a plan in paper format with a good indication of the location of the markers. The choice of the posts was made in order to have an optimal configuration with a good geometry allowing a good coverage of the site. This would allow the detection of possible problems of homogeneity on the accuracy of the points according to their location.

The main difficulties encountered are related to the intensity of the extractive activities on the site. All the markers that were located at the work sites and particularly in the excavated areas have disappeared. The work sites are the places where the extraction of minerals is taking place at a lightning speed, thus causing the loss of a significant number of landmarks. While, among these lost markers, there are some that were part of the initial choice for the observations, it was therefore necessary to replace them by others while maintaining the selection criteria. In addition, most of the posts that were in the fields were removed or relocated by the populations so as not to interfere with agricultural activities during the rainy season. The populations whose main activity is agriculture remove everything they find as bollards in their fields, either out of ignorance or by considering them as a threat of expropriation. The other difficulty was related to the fact that it has been decades since these markers were put in place and that in the meantime, many trees and shrubs have developed, they are bushy and constitute, in addition to the grass, real masks for many of the markers.

\subsection{GNSS Observations and Data Processing}

In this project, all observations were made using the differential positioning mode. This is the most accurate in GNSS because it eliminates systematic errors by performing differences in observations between stations [7].

The first observation session was for the placement of two support points (pivots), ICS1 and ICS2. To ensure maximum accuracy, we proceeded with the static positioning mode. The pivot was stationed on point B010 (second order point of RRS04) for a duration of $04 \mathrm{~h} 30$ corresponding to the duration of the observation session. The second receiver (mobile) occupied successively the points ICS1 and ICS2 for observations of more than one hour each. It should be noted that in static mode, the duration of the observations is very essential because the resolution of integer ambiguities systematically depends on it. In order to have the maximum precision on the coordinates of the stationary point, it is necessary to try to fix the whole ambiguities on all the measurements of phase carried out, reason for 
which, it is necessary a minimum number of measurements and thus a certain time of observation [8].

The raw data thus recorded were processed with the GNSS calculation software Leica Infinity 3.0 for baseline calculation.

Then, after the definition and materialization in the field of the two support points, the observation campaign was completed with raw measurements on six tie points and three control points which are respectively: 108, 112, 141, B18B, TN001, TN003 and 55, B18A, TN002. The coordinates of these points were determined by post-processing. For this purpose, three observation sessions were conducted. On 13/11/2020, we surveyed points 55, 108, and 112 between 13:46 and 16:00 from reference ICS2. On 11/14/2020, point 141 was determined with the base placed at ICS2 from 10:20 AM to 1:07 PM and then marker B18A was observed with the pivot stationed at reference ICS1 at 2:03 PM for 11 minutes. Finally, on $01 / 12 / 2020$, between $17: 42$ and $18: 37$ and in relation to the reference ICS1, a last session of observations allowed the determination of the coordinates of the terminals B18B, TN001, TN002 and TN003. The principle of measurements remains the same, the base stationed on ICS1 or ICS2 according to the closest to the point to be determined and the mobile placed successively on all nine landmarks for a long enough time (depending on the pivot-mobile distance) to record raw observations. For more details, we have presented in figure 1, the diagram of the observations made. The raw data were then processed with the Leica Infinity 3.0 software. After post-processing, the results obtained for the control points were not integrated in the calculation process of the adaptation parameters. This is to ensure their independence from the fitting parameters and to guarantee the reliability of the checks to be performed.

\section{Determination of the Transformation Parameters and Their Accuracy}

For the resolution of the problem and the optimization of the solution, we have opted for the least squares method. This method is the easiest to implement and the most used in metrological sciences [9]. For this, we first established in theory, the methodology and then proceeded to a simple numerical application.

\subsection{Methodology}

We want to determine $m$ unknown scalar quantities using $n$ observed quantities that are geometrically related to them by observation relations [10]. These observations can be direct or indirect. If they are direct, then they are independent, in addition to their value, we know their priori accuracy given by their root mean square $\sigma_{i}$ in this case the variance/covariance matrix on the observations is diagonal [10]. The inferred weighting matrix $P$ is then diagonal.

To translate the relationship between the parameters and the observations, we used the two-dimensional Helmert transformation. It expresses the relationship between the two datums which considered. In our case, this transformation is writ- 
ten in the vector form [11]:

$$
\left(\begin{array}{c}
E_{i} \\
N_{i}
\end{array}\right)=T+K R_{\theta}\left(\begin{array}{c}
X_{i} \\
Y_{i}
\end{array}\right)
$$

where:

$T$ is the translation vector of components $\left(T_{X}, T_{Y}\right)^{\mathrm{T}}$ between the ICS local datum and the RRS04,

$K$ is the scale factor (or homothetic ratio) between the two datums,

$R_{\theta}$ is the $2 \times 2$ rotation matrix to move from the local ICS local datum to the RRS04,

$\left(X_{i}, Y_{i}\right)$ the coordinates of point $P_{i}$ in the local system of ICS,

$\left(E_{i}, N_{i}\right)$ the coordinates of the same point $P_{i}$ in the RSS04.

By expanding, we obtain:

$$
\left(\begin{array}{c}
E_{i} \\
N_{i}
\end{array}\right)=\left(\begin{array}{c}
T_{X} \\
T_{Y}
\end{array}\right)+K\left(\begin{array}{cc}
\cos \theta & -\sin \theta \\
\sin \theta & \cos \theta
\end{array}\right)\left(\begin{array}{c}
X_{i} \\
Y_{i}
\end{array}\right)=\left(\begin{array}{c}
T_{X} \\
T_{Y}
\end{array}\right)+\left(\begin{array}{cc}
K \cos \theta & -K \sin \theta \\
K \sin \theta & K \cos \theta
\end{array}\right)\left(\begin{array}{c}
X_{i} \\
Y_{i}
\end{array}\right)
$$

Taking as auxiliary unknowns: $u=K \cos \theta$ and $v=K \sin \theta$, the system becomes:

$$
\left(\begin{array}{c}
E_{i} \\
N_{i}
\end{array}\right)=\left(\begin{array}{c}
T_{X} \\
T_{Y}
\end{array}\right)+\left(\begin{array}{cc}
u & -v \\
v & u
\end{array}\right)\left(\begin{array}{c}
X_{i} \\
Y_{i}
\end{array}\right)
$$

The unknowns $T_{X}, T_{Y}, u$ and $v$ will be determined by the least squares method using known common points in both systems [11].

Having $u$ and $v$, we can deduce $K=\sqrt{u^{2}+v^{2}}$ et $\theta=\tan ^{-1} \frac{v}{u}$.

Knowing that each known point $P_{i}$ in the two systems gives two equations:

$$
\begin{aligned}
& E_{i}=T_{X}+X_{i} u-Y_{i} v \\
& N_{i}=T_{Y}+Y_{i} u+X_{i} v
\end{aligned}
$$

Therefore, we need to know the coordinates of $p$ points $(p \geq 2)$ in both systems in order to solve the following $n \times 4$ matrix system (where $n=2 p \geq 4$ ):

$$
\left(\begin{array}{c}
E_{1} \\
N_{1} \\
E_{2} \\
N_{2} \\
\vdots \\
E_{n} \\
N_{n}
\end{array}\right)=\left(\begin{array}{cccc}
1 & 0 & X_{1} & -Y_{1} \\
0 & 1 & Y_{1} & X_{1} \\
1 & 0 & X_{2} & -Y_{2} \\
0 & 1 & Y_{2} & X_{2} \\
\vdots & \vdots & \vdots & \vdots \\
1 & 0 & X_{n} & -Y_{n} \\
0 & 1 & Y_{n} & X_{n}
\end{array}\right)\left(\begin{array}{c}
T_{X} \\
T_{Y} \\
u \\
v
\end{array}\right)
$$

In the least squares optimization, we take into account the accidental measurement errors that must be added to the Equation (5).

For more clarity, we will first give in the following lines, some notations before giving the rigorous observation relations, taking into account the measurement residues.

Let be: 


$$
L=\left(\begin{array}{c}
E_{1} \\
N_{1} \\
E_{2} \\
N_{2} \\
\vdots \\
E_{n} \\
N_{n}
\end{array}\right), \quad A=\left(\begin{array}{cccc}
1 & 0 & X_{1} & -Y_{1} \\
0 & 1 & Y_{1} & X_{1} \\
1 & 0 & X_{2} & -Y_{2} \\
0 & 1 & Y_{2} & X_{2} \\
\vdots & \vdots & \vdots & \vdots \\
1 & 0 & X_{n} & -Y_{n} \\
0 & 1 & Y_{n} & X_{n}
\end{array}\right), \quad V=\left(\begin{array}{c}
V_{E_{1}} \\
V_{N_{1}} \\
V_{E_{2}} \\
V_{N_{2}} \\
\vdots \\
V_{E_{n}} \\
V_{N_{n}}
\end{array}\right), \quad X=\left(\begin{array}{c}
T_{X} \\
T_{Y} \\
u \\
v
\end{array}\right)
$$

$L$ is the vector of observations,

$A$ is the matrix of coefficients,

$V$ is the vector of accidental measurement errors (or vector of measurement residues),

$X$ is the vector of unknowns.

Then the matrix Equation (5) can be written:

$$
L=A X
$$

Equation (5a) expresses the theoretical relationship that exists between the two datums without taking into account measurement errors. This relationship is therefore the one that would exist between the two datums if all measurements were perfect, without errors. Now, to obtain the least squares equation, we must take into account the errors made in the practical realization of the measurements. This equation is obtained by adding to Equation (5a), the vector $V$ of the accidental errors of measurement.

The least squares equation is then written:

$$
A X=L+V
$$

Since all measurements were performed under the same conditions and independently, the priori accuracy is considered to be the same $\left(\sigma_{i}=\sigma, 1 \leq i \leq n\right)$ and, in addition, the weight matrix $P$ is diagonal. In this present case, we can choose $P=$ $I$, the identity matrix. The least squares condition $V^{\text {T }} P V$ minimum becomes $(A X-L)^{\mathrm{T}}(A X-L)$ minimum or by expanding, $X^{\mathrm{T}} A^{\mathrm{T}} A X-2 X^{\mathrm{T}} A^{\mathrm{T}} L+L^{\mathrm{T}} L$ minimum. Let the scalar function be:

$F(X)=X^{\mathrm{T}} A^{\mathrm{T}} A X-2 X^{\mathrm{T}} A^{\mathrm{T}} L+L^{\mathrm{T}} L$. We show that solving for min $F(X)$ implies $d F(X)=0$, which gives the following normal system:

$$
N X=C
$$

where: $N=A^{\mathrm{T}} A$ and $C=A^{\mathrm{T}} L$.

Equation (7) is also written:

$$
A^{\mathrm{T}} V=0
$$

Equation (7a) is called the normalization equation of the least squares solution.

The least squares solution $\hat{X}=\left(\begin{array}{c}\hat{T}_{X} \\ \hat{T}_{Y} \\ \hat{u} \\ \hat{v}\end{array}\right)$, is given by: 
$\hat{X}=N^{-1} C=\left(A^{\mathrm{T}} A\right)^{-1}\left(A^{\mathrm{T}} L\right)$.

The vector of residuals of the measurements, $\hat{V}=L-A \hat{X}$ is an estimator of the posteriori errors related to the observations. From this vector of residuals, we give an estimate of the unit variance factor $s_{0}^{2}$, noted $\hat{s}_{0}^{2}$ and defined by:

$$
\hat{s}_{0}^{2}=\frac{\hat{V}^{\mathrm{T}} \hat{V}}{n-4} .
$$

The posteriori accuracy of a component $\hat{X}_{i}$ of the optimized solution $\hat{X}$, noted $S_{0} \hat{\omega}_{i}$ is given by:

$$
S_{0} \hat{\omega}_{i}=\hat{s}_{0} \sqrt{N_{i i}^{-1}}
$$

where $N_{i i}^{-1}$ is the $I^{\text {th }}$ diagonal element of the cofactor matrix $N^{-1}$.

\subsection{Numerical Application}

In our case, we have the following input data:

$$
A=\left(\begin{array}{cccc}
1 & 0 & 105332.010 & -94146.860 \\
0 & 1 & 94146.860 & 105332.010 \\
1 & 0 & 105244.900 & -93625.460 \\
0 & 1 & 93625.460 & 105244.900 \\
1 & 0 & 104053.600 & -91429.190 \\
0 & 1 & 91429.190 & 104053.600 \\
1 & 0 & 102680.776 & -102047.503 \\
0 & 1 & 102047.503 & 102680.776 \\
1 & 0 & 104708.691 & -93583.373 \\
0 & 1 & 93583.373 & 104708.691 \\
1 & 0 & 104935.450 & -93227.892 \\
0 & 1 & 93227.892 & 104935.450
\end{array}\right) \text { et } L=\left(\begin{array}{c}
307073.9107 \\
1663409.1995 \\
306982.1466 \\
1662888.6000 \\
305771.3462 \\
1660702.9764 \\
304493.0737 \\
1671333.0846 \\
306445.5407 \\
1662851.2442 \\
306669.1247 \\
1662493.7596
\end{array}\right) .
$$

Before entering their coordinates in the matrix $A$, the points were first analyzed by comparing their corresponding distances. For two given points, the corresponding distances are the distance separating them according to the local datum and the distance separating them according to RRS04. The points used in the construction of the matrix $A$ all gave, on average, satisfactory differences between their corresponding distances, compared to the tolerance that was set. We built and executed the calculation algorithm that has already been theoretically established for the calculations.

After the calculations, the results obtained are presented in the following lines.

The resolution of the normal system $N X=C$ requires the knowledge of the matrices:

$$
N^{-1}=\left(\begin{array}{cccc}
266.8863 & 0.0000 & -0.0014 & 0.0013 \\
0.0000 & 266.8863 & -0.0013 & -0.0014 \\
-0.0014 & -0.0013 & 0.0000 & 0.0000 \\
0.0013 & -0.0014 & 0.0000 & 0.0000
\end{array}\right) \text { et } C=\left(\begin{array}{c}
1837435.1426 \\
9983678.8643 \\
1137294761986.8700 \\
869257291149.5500
\end{array}\right) \text {. }
$$

The optimized solution, thus deduced, is presented in Table 1, with its preci- 
sion calculated from $\hat{s}_{0}^{2}=0.0007$ and the cofactor matrix $N^{-1}$ (Table 1). The unit factor $\hat{s}_{0}^{2}$ is estimated from the vector $V$ of the measurement residuals.

The accuracy on the $T_{X}$ and $T_{Y}$ parameters could be related to the order of magnitude of these parameters which shows the distance of the center of the local datum from that of the system associated with RRS04. It could also be linked to the degree of internal coherence of the local system. But it could also be due to the difference in accuracy between the two datums which used.

\section{Validation}

After the estimation of the parameters, we proceeded to the control and validation of the results taking into account the specifications. For this purpose, we determined the vector of residuals of the measurements estimating the errors on the observations (Table 2). We also calculated the standard deviation of the measurement errors, denoted $\sigma$. This is a global estimator of the precision of the measurements and is calculated on the basis of the residuals as follows:

$$
\sigma=\sqrt{\frac{\sum V_{i}^{2}}{n}}
$$

where $n$ is the number of observations and for our case $n=12$.

Table 1. Migration parameters and their accuracy.

\begin{tabular}{ccc}
\hline Parameters & Value & Accuracy (cm) \\
\hline$T_{X}$ & $200,908.273$ & 42.60 \\
$T_{Y}$ & $1,570,203.594$ & 42.60 \\
$u$ & 1 & 0 \\
$V$ & -0.009 & 0 \\
\hline
\end{tabular}

Table 2. Measurement residuals and accuracy $\sigma$ of the attachment.

\begin{tabular}{|c|c|c|c|}
\hline Terminals & Coordinates & Residuals $\left(V_{i}\right)(\mathrm{cm})$ & $\sigma(\mathrm{cm})$ \\
\hline \multirow{2}{*}{108} & $\mathrm{E}$ & 3.129 & \multirow{12}{*}{ \pm 2.129} \\
\hline & $\mathrm{N}$ & 2.439 & \\
\hline \multirow{2}{*}{112} & $\mathrm{E}$ & 1.401 & \\
\hline & $\mathrm{N}$ & 2.835 & \\
\hline \multirow{2}{*}{141} & $\mathrm{E}$ & 1.150 & \\
\hline & $\mathrm{N}$ & -1.722 & \\
\hline \multirow{2}{*}{$\mathrm{B} 18 \mathrm{~B}$} & $\mathrm{E}$ & 0.560 & \\
\hline & $\mathrm{N}$ & -0.685 & \\
\hline \multirow{2}{*}{ TN001 } & $\mathrm{E}$ & -3.019 & \\
\hline & $\mathrm{N}$ & -1.434 & \\
\hline \multirow{2}{*}{ TN003 } & $\mathrm{E}$ & -3.221 & \\
\hline & $\mathrm{N}$ & -1.432 & \\
\hline
\end{tabular}


Table 3. Comparison of the calculated coordinates with the measured coordinates of the control points.

\begin{tabular}{ccccccc}
\hline \multirow{2}{*}{ Terminals } & \multicolumn{2}{c}{ Measured coordinates } & \multicolumn{2}{c}{ Calculated coordinates } & \multicolumn{2}{c}{ 1 Differences 1 } \\
\cline { 2 - 7 } & $\mathrm{E}$ & $\mathrm{N}$ & $\mathrm{E}$ & $\mathrm{N}$ & $\mathrm{E}$ & $\mathrm{N}$ \\
\hline 55 & $301,960.845$ & $1,666,437.370$ & $301,960.803$ & $1,666,437.347$ & 4.12 & 2.23 \\
B18A & $304,541.436$ & $1,671,334.298$ & $304,541.417$ & $1,671,334.296$ & 1.96 & 0.21 \\
TN002 & $306,546.982$ & $1,662,683.354$ & $306,547.001$ & $1,662,683.368$ & 1.84 & 1.40 \\
\hline
\end{tabular}

We can see that the overall accuracy $\sigma$, which is that of the attachment is good compared to the project specifications which impose an accuracy of $\pm 5 \mathrm{~cm}$. Even if it is rounded to be more constraining, it will be equal to $\pm 3 \mathrm{~cm}$, clearly better than $\pm 5 \mathrm{~cm}$.

We also performed a check of our results by comparing the calculated coordinates from the parameters and the measured coordinates of the control points. The results of this comparison are presented in the following Table (Table 3).

Table 3 shows that for each control point, the calculated coordinates are within the tolerances. For each point and for each component, the difference in absolute value between the calculated value and the observed value is less than $5 \mathrm{~cm}$. In addition, the differences in coordinates found validate the result of the residual measurements. Overall, the results of the control are in line with the accuracy of the measurements and that of the attachment.

\section{Conclusions}

The GNSS measurement technique, with its relative positioning mode, in combination with the least squares method has allowed us today to determine the fitting parameters and to directly tie the local ICS grid to the RRS04 with an accuracy of $\pm 3 \mathrm{~cm}$. This technique gave us the possibility to avoid long paths with the total station. The measurements made are direct and therefore free of possible error accumulation related to intermediate observations. Thus, the parameters of passage between the local datum and the RRS04 are clearly defined. From now on, the ICS have a calculation algorithm to carry out the process of simple and fast passage.

With a standard deviation lower than the tolerance of $\pm 5 \mathrm{~cm}$ set by the ICS for this project, all the marks of the canvas are now attached to the RRS04 with a satisfactory accuracy. The ICS now have a new terrestrial reference system based on RRS04 which is the solution to the constraints and limitations associated with the use of a local datum.

Thus, the recommendation by the competent authorities in the new mining code to actors in the mining sector to use the national terrestrial reference system (RRS04) as a reference is now applicable by the ICS. The latter can now work with more accurate and reliable coordinates of a homogeneous and more global system to ensure national and international consistency of their spatial data. Such data allows the company to be more competitive and to easily exchange data with 
its collaborators. It also facilitates the audit missions with the industry because the auditors have expressed the wish to work in the future with UTM coordinates in the RRS04.

Obtaining the parameters and changing the system is the first phase of the project. The migration process will be complete once all existing localizable data in the ICS is redefined to RRS04. To do this, all that is required now is processing of this data involving transformations from the parameters. This will allow the topographic plans and maps and the geological maps to be readapted. The topographic profiles as well as the cross-sections showing the production runs will therefore be redefined in relation to the new reference system. The new reference system is now ready to be used as a reference for the company's geospatial data, especially for the mining and geology division.

In perspective, the voluminous size of the spatial data produced by ICS is not an easy task in terms of storage and processing. The management of such a flow of information is very delicate. It would therefore be very interesting for the ICS, with the connection to the national datum (RRS04), to set up a geographic information system (GIS) for more efficiency in the work. The purpose of GIS is to allow true spatial data management (this is the key point that differentiates a GIS software from a CAD software), and to serve as a decision support through a priori much more powerful management capability than in the case of paper data [12]. This GIS with an integrated Database Management System (DBMS), would be a powerful tool for storing, accessing, analyzing spatial data, disseminating and sharing the results obtained for easy and timely decision making by CSI managers. It would allow for easier monitoring of the mine and more effective planning of mining operations.

\section{Conflicts of Interest}

The authors declare no conflicts of interest regarding the publication of this paper.

\section{References}

[1] Décret $n^{\circ} 2017-459$ fixant les modalités d'application de la loi $n^{\circ} 2016-32$ du 08 novembre 2016 portant code minier. $37 \mathrm{p}$.

[2] Loi n ${ }^{\circ} 2016-32$ du 08 novembre 2016 portant code minier. $53 \mathrm{p}$.

[3] Diakite, D. (1988) Rapport sur les travaux topographiques du panneau II de Keur Mor FALL. CSPT-Division Mine. 29 p.

[4] Diouf, D. (2019) Etat actuel du Sénégal en matière de GNSS et systèmes de reference. $11 \mathrm{p}$.

[5] IGN-France International; DTGC (2004) Rapport de calcul du réseau de référence du Sénégal (RRS04). 57 p.

[6] Moritz, H. (2000) Geodetic Reference System 1980. Journal of Geodesy, 74, 128-134. https://doi.org/10.1007/s001900050278

[7] Bosser, P. (2012). GNSS: Systèmes globaux de positionnement par satellite. Ecole Nationale des Sciences Géographiques (ENSG, IGN)-Département Positionnement 
Terrestre et Spatial. $113 \mathrm{p}$.

[8] Legros, R., Morel, L., Viguier, F. and Birot, F. (2013) Méthodes de travail dans les réseaux GNSS. Geodata Diffusion, ESGT, SNCF. 78 p.

[9] Diouf, D. and Durand, S. (2018). Cours de Calcul d'erreurs et compensation-M2. 99 p.

[10] Ben Hadj Salem, A. (2017) Eléments de Géodésie et de la Théorie des Moindres Carrés pour les Elèves Ingénieurs Géomaticiens. Edité par Nour-Publishing. 365 p.

[11] Ben Hadj Salem, A. (2015) Cours de Cartographie Mathématique et les Transformations de Passage entre les Systèmes Géodésiques. Cours enseigné à l'Ecole Supérieure Privée d'Aéronautique et des Technologies de Tunis (ESAT). 86 p. https://vixra.org/pdf/1511.0219v1.pdf

[12] Kasser, M. (2013) Topographie, topométrie, géodésie. Techniques de l'ingénieur, C 5010v2, 20 p. 